Check for updates

Cite this: RSC Adv., 2017, 7, 41304

Received 29th June 2017

Accepted 15th August 2017

DOI: 10.1039/c7ra07220a

rsc.li/rsc-advances

\section{Preparation and optical properties of magnetic carbon/iron oxide hybrid dots}

\author{
Yin Hu, ${ }^{\text {ab }}$ Ping Wang, ${ }^{\mathrm{b}}$ Christopher E. Bunker, ${ }^{\mathrm{c}}$ Lindsay R. Teisl, ${ }^{\mathrm{b}}$ Mclver Reibold, ${ }^{\mathrm{b}}$ \\ Sijia Yan, ${ }^{b}$ Haijun Qian, ${ }^{b}$ Dawei $\mathrm{He}^{\mathrm{a}}$ and Ya-Ping Sun (D)*b
}

Carbon dots (CDots), generally defined as small carbon nanoparticles with various surface passivation schemes for bright and colorful fluorescence emissions, have emerged to represent a rapidly advancing and expanding research field. Building upon the basic structural configuration of CDots, iron oxides were introduced for both magnetic and fluorescence properties in the resulting hybrid dots, thus resulting in more capabilities beyond those of neat CDots. The carbon $/ \mathrm{Fe}_{3} \mathrm{O}_{4}$ hybrid dots with oligomeric polyethylene glycol or polyethyleneimine for surface functionalization and passivation were prepared in a facile thermal carbonization synthesis using microwave energy, coupled with magnetic separation. The magnetic hybrid dots were fluorescent over the visible spectrum, but the fluorescence quantum yields were found to be lower than those of their neat CDots counterparts, for which a possible quenching effect due to the $\mathrm{Fe}_{3} \mathrm{O}_{4}$ in the dot structure was probed and evaluated. The results support the notion that the hybrid dot configuration could serve as a platform for imparting magnetic properties into brightly fluorescent CDots. Also investigated were the dot structures and compositions to gain a rough view of the carbon-iron oxide configurations in the hybrid dots. The application potential of the hybrid dots and opportunities for their further improvements are discussed.

\section{Introduction}

Carbon is commonly perceived as being a dark-colored matter, with little attention paid to its optical properties other than its use as a blackbody for photon to heat conversion. At the nanoscale, however, carbon materials have exhibited rich optical properties, including effective photon harvesting to drive excited state energy and charge transfer processes, which are widely pursued for a variety of applications. ${ }^{1-3}$ Especially, carbon "quantum" dots or carbon dots (CDots, Fig. 1) ${ }^{4}$ have emerged to represent a new class of carbon-based optical nanomaterials with bright and colorful fluorescence emissions, attracting much attention recently. ${ }^{5-13}$ In fact, the investigation on the science and technology of CDots has become a rapidly advancing and expanding research field, as made evident by the large number of publications in the literature. ${ }^{12,13}$

CDots are generally defined as small carbon nanoparticles with various surface passivation schemes, among which the use of organic molecules or species has been effective (Fig. 1).4,14,15

${ }^{a}$ Key Laboratory of Luminescence and Optical Information, Ministry of Education, Institute of Optoelectronic Technology, Beijing Jiaotong University, Beijing 100044, China

${ }^{b}$ Department of Chemistry and Laboratory for Emerging Materials and Technology, Clemson University, Clemson, South Carolina 29634-0973, USA. E-mail: syaping@ clemson.edu

${ }^{c}$ Propulsion Directorate, Air Force Research Laboratory, Wright-Patterson Air Force Base, Ohio 45433, USA
Beyond the neat CDots, the same structural configuration, along with some of the synthesis methods for CDots, has been used as the basis for carbon-based hybrid nanostructures with semiconductors including $\mathrm{TiO}_{2}$ and others for purposes such as much improved optical properties ${ }^{16,17}$ and enhanced photocatalytic
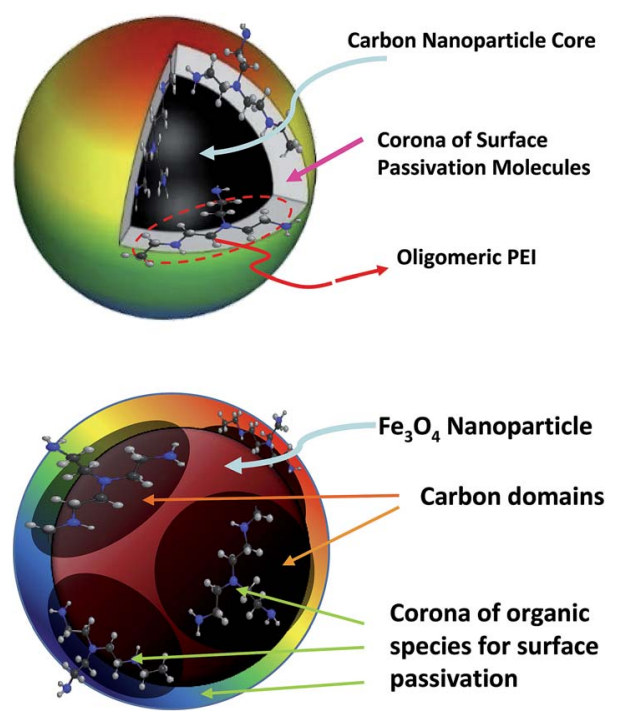

Fig. 1 A cartoon illustration on a CDot with polyethyleneimine (PEI) for surface functionalization (upper) ${ }^{45}$ and a carbon $/ \mathrm{Fe}_{3} \mathrm{O}_{4}$ hybrid dot with organic species for the desired passivation effect (lower). 
activities. ${ }^{18-22}$ Similarly, unpaired electrons have been introduced into the structure of CDots to impart magnetic properties in the resulting hybrid nanostructures, with far-reaching implications in terms of an understanding of the effects and/or interactions between the optical and magnetic properties and in the expanded development of CDots-derived technologies. ${ }^{23-32}$ For example, Du, et al. fabricated Gd-doped CDots by hydrothermal treatment of a mixture of glycine and gadopentetic acid, and found that these dots could serve as $T_{1}$ contrast agents in magnetic imaging, exhibiting also the dose enhancement effect in inhibiting the growth of solid tumor. ${ }^{30}$ $\mathrm{Han}$, et al. used the solvothermal reaction of $\mathrm{MnCl}_{2}, \mathrm{EDTA}$, and triethylenetetramine in ethylene glycol for the preparation of Mn-doped CDots, and evaluated the dots thus prepared in MRI-fluorescence dual-modality bioimaging. ${ }^{28}$ In a similar solvothermal synthesis, Wang, et al. prepared porous carbon/ $\mathrm{Fe}_{3} \mathrm{O}_{4}$ hybrid nanoparticles of large sizes (around $100 \mathrm{~nm}$ in diameter) for their serving as vehicles to carry anti-cancer drug doxorubicin and for the function of light-controlled drug release. $^{32}$

In fact, carbon/metal oxide hybrid dots may represent a versatile platform for combining optical properties with magnetic and other functionalities, alternative to the widely pursued strategies such as incorporating fluorescent dye molecules, noble metal nanoparticles, and/or semiconductor quantum dots into polymeric or silica composite nanospheres. $^{32,33}$ For those materials, a significant amount of results have been reported in the literature on their various promising potential applications, such as biomedical imaging, drug delivery and controlled release, and other uses. ${ }^{34-39}$ Among a few available studies of nanoscale $\mathrm{Fe}_{3} \mathrm{O}_{4}$ as the metal oxide in hybrid dots involving carbon, $\mathrm{Li}$, et al. fabricated carbon/ $/ \mathrm{Fe}_{3} \mathrm{O}_{4}$ nanoparticles of relatively small sizes for their uses in cell imaging by both magnetic and fluorescence modalities, though the optical excitation and fluorescence emissions of these nanoparticles were mostly limited to the UV spectral region..$^{33}$

In this work, carbon $/ \mathrm{Fe}_{3} \mathrm{O}_{4}$ hybrid dots with oligomeric polyethylene glycol (PEG) or polyethyleneimine (PEI) for surface functionalization and passivation were prepared in a facile synthesis based on thermal carbonization with microwave energy, coupled with magnetic separation. The hybrid dots were characterized for an understanding of their structures and compositions, with the results suggesting that the hybrid dot structure may be viewed as a relatively small $\mathrm{Fe}_{3} \mathrm{O}_{4}$ nanoparticle decorated with carbon moieties in very roughly a core-shell like configuration, functionalized by the organic species (Fig. 1). Optical absorption and fluorescence properties of the hybrid dots were compared with those of their neat CDots counterparts, and an emphasis of the investigation was on probing any quenching effect of the $\mathrm{Fe}_{3} \mathrm{O}_{4}$ on the fluorescence properties of the passivated carbon domains in the hybrid dots. The potential and also challenges in the development of magnetic carbon/metal oxide hybrid dots with high optical performance (bright and colorful fluorescence emissions in particular) are highlighted and discussed.

\section{Experimental section}

\subsection{Materials}

Ferric ammonium citrate $\left(\mathrm{C}_{6} \mathrm{H}_{8} \mathrm{O}_{7} \cdot x \mathrm{Fe}^{3+} \cdot y \mathrm{NH}_{3}\right)$ was purchased from VWR, ferrous sulfate heptahydrate $\left(\mathrm{FeSO}_{4} \cdot 7 \mathrm{H}_{2} \mathrm{O}\right)$ from J. T. Baker, and potassium thiocyanate (KSCN) from Strem Chemicals, Inc. Oligomeric polyethylene glycol ( $\mathrm{PEG}_{1500}$, average molecular weight $\sim 1500$ ) was obtained from ACROS Organics, polyethylenimine (PEI, branched, average molecular weight $\sim 1200$ ) from Polyscience, Inc., and polyvinylpyrrolidone (PVP, average molecular weight $\sim 40$ 000) from Sigma Aldrich. Silicon carbide (120 grit) was acquired from Panadyne Abrasives, nitric acid and sulfuric acid from Fisher Chemical, and potassium permanganate from ACROS. Dialysis membrane tubings (molecular weight cut-off $\sim 500$ and $\sim 1000$ ) were supplied by Spectrum Laboratories. Water was deionized and purified by being passed through a Labconco WaterPros water purification system.

\subsection{Measurements}

Powder X-ray diffraction measurements were performed on a Rigaku Ultima IV X-ray diffractometer with $\mathrm{Cu} \mathrm{K} \alpha$ radiation $(\lambda=1.5418 \AA)$ at $25^{\circ} \mathrm{C}$. The calculation of crystallite sizes based on the Scherrer equation was enabled by the PDXL 1.8.1.0 software. The transmission electron microscopy (TEM) imaging was carried out on a Hitachi H-9500 high-resolution TEM system. The TEM specimen was prepared by depositing a few drops of a dilute sample solution onto a copper grid, followed by careful evaporation to remove the solvent. UV/vis absorption spectra were recorded on a Shimadzu UV2501-PC spectrophotometer. Fluorescence spectra were acquired on a Jobin-Yvon emission spectrometer equipped with a $450 \mathrm{~W}$ xenon source, Gemini-180 excitation and Triax-550 emission monochromators, and a photon counting detector (Hamamatsu R928P PMT at $950 \mathrm{~V}$ ). 9,10-Bis(phenylethynyl)-anthracene in cyclohexane was used as a standard in the determination of fluorescence quantum yields by the relative method (matching the absorbance at the excitation wavelength between the sample and standard solutions and comparing their corresponding integrated total fluorescence intensities). FT-IR spectra were measured on a Shimadzu IRAffinity-1S spectrophotometer with the Single Reflection ATR accessory for solid samples.

\subsection{PEGylated carbon/ $/ \mathrm{Fe}_{3} \mathrm{O}_{4}$ hybrid dots}

Ferric ammonium citrate $(0.5 \mathrm{~g})$ and $\mathrm{PEG}_{1500}(1 \mathrm{~g})$ were mixed in water $(10 \mathrm{~mL})$ in a small scintillation vial with stirring to yield a brown-colored solution, followed by the removal of water via purging with nitrogen gas for the reactant mixture. Separately, a silica crucible casting dish (about $8 \mathrm{~cm}$ in diameter and $2.5 \mathrm{~cm}$ in height) containing silicon carbide (150 g) was pre-heated in a conventional microwave oven at $500 \mathrm{~W}$ for $3 \mathrm{~min}$. The small scintillation vial containing the reactant mixture was immersed in the pre-heated silicon carbide bath for processing in the same microwave oven. The initial treatment was at $1000 \mathrm{~W}$ until smoke started to appear (after about half min). The oven was opened, and the vial containing the mixture was taken out of the 
silicon carbide bath to be in the ambient for about $1 \mathrm{~min}$. Then, the vial was re-immersed in the silicon carbide bath for a repeat of the same cycle of microwave treatment until smoke appearing and then out in the ambient for about $1 \mathrm{~min}$. After a total of 7 treatment cycles, the reaction mixture was collected and cooled to the ambient temperature, and then dispersed in deionized water $(10 \mathrm{~mL})$ with vigorous sonication. The resulting aqueous dispersion was allowed to stand for about $10 \mathrm{~min}$ before the separation in a magnetic separator (Dynal MPC-L). For the separation, the aqueous dispersion of the reaction mixture was in the separator for about $2 \mathrm{~h}$. The supernatant was removed, and the magnetic precipitate was washed with water and precipitated again in the magnetic separator. After the repeated washing until the supernatant became colorless, the final magnetic precipitate was collected as the as-prepared sample of the PEGylated carbon/ $\mathrm{Fe}_{3} \mathrm{O}_{4}$ hybrid dots (PEG-C/ $\mathrm{Fe}_{3} \mathrm{O}_{4}$-dots).

\subsection{Polyethylenimine-functionalized carbon/ $/ \mathrm{Fe}_{3} \mathrm{O}_{4}$ hybrid dots}

Ferric ammonium citrate $(0.5 \mathrm{~g})$ and polyethylenimine (PEI, $1 \mathrm{~g}$ ) were mixed in water $(10 \mathrm{~mL})$ in a small scintillation vial with stirring to yield a brown-colored solution, followed by the removal of water via purging with nitrogen gas for the reactant mixture. Separately, a silica crucible casting dish (about $8 \mathrm{~cm}$ in diameter and $2.5 \mathrm{~cm}$ in height) containing silicon carbide (150 g) was pre-heated in a conventional microwave oven at $500 \mathrm{~W}$ for $3 \mathrm{~min}$. The small scintillation vial containing the reactant mixture was immersed in the pre-heated silicon carbide bath for processing in the same microwave oven. The processing was in multiple cycles, each of which consisted of the treatment by microwave at $400 \mathrm{~W}$ until smoke started to appear in the sample vial, taking the vial out of the silicon carbide bath to be in the ambient for about $1 \mathrm{~min}$, and then reimmersing the vial in the silicon carbide bath for the next cycle. After a total of 12, the reaction mixture was collected and cooled to the ambient temperature, and then dispersed in deionized water $(10 \mathrm{~mL})$ with vigorous sonication. The resulting aqueous dispersion was allowed to stand for about $10 \mathrm{~min}$ before the magnetic separation by using the same protocol described above to collect the final magnetic precipitate as the as-prepared sample of the PEI-functionalized carbon/ $\mathrm{Fe}_{3} \mathrm{O}_{4}$ hybrid dots (PEI-C/ $/ \mathrm{Fe}_{3} \mathrm{O}_{4}$-dots).

\subsection{Iron content and removal}

Iron contents in the hybrid dots were quantified by using the classical colorimetric method with the use of a thiocyanate agent for the formation of red-colored iron(III) complex. For the standard curve, an aqueous solution of $\mathrm{FeSO}_{4} \cdot 7 \mathrm{H}_{2} \mathrm{O}(0.24895 \mathrm{~g}$ in $50 \mathrm{~mL}$ ) was prepared, and to the solution was added concentrated $\mathrm{H}_{2} \mathrm{SO}_{4}(2.5 \mathrm{~mL})$ and then aqueous $\mathrm{KMnO}_{4}(2 \%)$ dropwise until the purple color in the solution stopped fading. The resulting solution was diluted to $500 \mathrm{~mL}$ as the standard stock solution. An aliquot of the standard stock solution $(10 \mathrm{~mL})$ was diluted to $100 \mathrm{~mL}$ as the standard working solution. Five $25 \mathrm{~mL}$ volumetric flasks were used, to which different volumes of the working solution $(0,0.5,1.0,2.0$, and $3.0 \mathrm{~mL})$ were added, and then water $(5 \mathrm{~mL}), \mathrm{H}_{2} \mathrm{SO}_{4}(50 \%, 1 \mathrm{~mL}), \mathrm{KMnO}_{4}$ $(1 \%, 20 \mu \mathrm{L})$, and $\mathrm{KSCN}(20 \%, 2 \mathrm{~mL})$ were added to each flask, followed by the dilution with water to the full volume of each flask $(25 \mathrm{~mL})$. The absorbances of the solutions at $470 \mathrm{~nm}$ were measured for the standard curve.

For the digestion before the colorimetric analysis, a dot sample was refluxed in $\mathrm{HNO}_{3}(20 \%, 25 \mathrm{~mL})$ for $2 \mathrm{~h}$, and then the solution was concentrated to less than $10 \mathrm{~mL}$ in volume, followed by being cooled to room temperature and diluted to 10 $\mathrm{mL}$ for the working solution of the sample. The working solution ( $2 \mathrm{~mL}$ ) was added to a $25 \mathrm{~mL}$ volumetric flask, and to the flask were then added water $(5 \mathrm{~mL}), \mathrm{H}_{2} \mathrm{SO}_{4}(50 \%, 1 \mathrm{~mL}), \mathrm{KMnO}_{4}$ $(1 \%, 20 \mu \mathrm{L})$ and $\mathrm{KSCN}(20 \%, 2 \mathrm{~mL})$, followed by the dilution with water to the full volume of the flask $(25 \mathrm{~mL})$. The absorbance of the solution at $470 \mathrm{~nm}$ was measured, and the iron content in the sample was obtained from the standard curve.

For the removal of $\mathrm{Fe}_{3} \mathrm{O}_{4}$ from the hybrid dots, a dot sample in aqueous dispersion ( $2 \mathrm{~mL}$ ) was mixed with $\mathrm{HCl}(20 \%, 25 \mathrm{~mL})$, and the mixture was refluxed for $30 \mathrm{~min}$. The reaction mixture was cooled back to ambient temperature, and dialyzed (molecular weight cutoff $\sim 500$ ) against fresh water for $12 \mathrm{~h}$. The solution in the dialysis tubing was collected and concentrated for characterization and investigations.

\section{Results and discussion}

The PEGylated hybrid dots $\mathrm{PEG}-\mathrm{C} / \mathrm{Fe}_{3} \mathrm{O}_{4}$-dots were prepared in the one-pot thermal carbonization processing of precursor mixtures containing oligomeric PEGs $\left(\mathrm{PEG}_{1500}\right)$ and ferric ammonium citrate (FAC) in various PEG/FAC mass ratios. Different processing conditions involving the use of microwave irradiation for the thermal carbonization were evaluated, including various combinations of microwave power and irradiation time duration in a single treatment and the use of multiple treatment cycles, each of which consisted of microwave heating and then a quick cooling in the ambient. The reaction mixture from each processing was dispersed in water, and the resulting aqueous dispersion was separated in terms of magnetic precipitation in a magnetic separator with a permanent magnet to harvest the magnetically responsive fraction. The evaluation of the outcomes associated with different PEG/ FAC mass ratios and thermal carbonization conditions was based on parameters including the product yield for the dot sample, the aqueous dispersibility, and the $\mathrm{C} / \mathrm{Fe}$ ratio in the sample from the magnetic separation. The use of a PEG/FAC ratio of 2 in the precursor mixture coupled with multiple-cycle microwave treatments was identified as being more desirable for the carbonization processing. Briefly on the multiple treatment cycles for the thermal carbonization with microwave irradiation, in the initial cycle the precursor FAC-PEG mixture was immersed in a pre-heated silicon carbide bath for heating in a conventional microwave oven until the onset of visible smoking in the sample vial, and then the vial was taken out of the oven for a quick cooling in the ambient, followed immediately by the next treatment cycle of the same microwave irradiation and quick cooling. At the conclusion of the thermal carbonization processing, the targeted hybrid dots 
PEG-C/ $\mathrm{Fe}_{3} \mathrm{O}_{4}$-dots could readily be harvested as the magnetically responsive fraction in the magnetic separation of the reaction mixture.

Similarly, the PEI-functionalized hybrid dots $\mathrm{PEI}-\mathrm{C} / \mathrm{Fe}_{3} \mathrm{O}_{4}$ dots were prepared by thermal carbonization processing of FAC-PEI mixtures in terms of the same multiple microwave treatment - quick cooling cycles, and isolated from the reaction mixture as the magnetically responsive fraction in a magnetic separation.

Powder X-ray diffraction measurements were used for the characterization of the hybrid dots, and the diffraction patterns were found to be due exclusively to $\mathrm{Fe}_{3} \mathrm{O}_{4}$ (Fig. 2), suggesting that the samples were free from any meaningful presence of other crystalline species, and the carbon domains in the hybrid dots were largely amorphous, as commonly observed for core carbon nanoparticles in CDots. ${ }^{3,40-42}$ All of the diffraction peaks ( $2 \theta$ of $111,220,311,400,422,511$, and 440 , Fig. 2) were broadened, consistent with the nanoscale nature of the $\mathrm{Fe}_{3} \mathrm{O}_{4}$ in the hybrid dots. With the peak broadening, calculations based on the Scherrer equation yielded average $\mathrm{Fe}_{3} \mathrm{O}_{4}$ nanocrystal sizes of $10 \pm 1 \mathrm{~nm}$ and $9.5 \pm 0.9 \mathrm{~nm}$ in the $\mathrm{PEG}-\mathrm{C} / \mathrm{Fe}_{3} \mathrm{O}_{4}$-dots and $\mathrm{PEI}-\mathrm{C} / \mathrm{Fe}_{3} \mathrm{O}_{4}$-dots, respectively. The size estimates were supported by the TEM imaging results. Shown in Fig. 3 is a representative TEM image for the PEI-C/ $/ \mathrm{Fe}_{3} \mathrm{O}_{4}$-dots. Because of the high Z-contrast between carbon and iron, the size profiles in TEM images are dominated by the $\mathrm{Fe}_{3} \mathrm{O}_{4}$ nanoparticles in the hybrid dots. The statistical analysis based on multiple TEM images yielded an average size of $9.7 \mathrm{~nm}$ and a size distribution standard deviation of about $2 \mathrm{~nm}$ (Fig. 3), consistent with the estimate based on the Scherrer equation.

FT-IR spectra of the PEG-C/ $/ \mathrm{Fe}_{3} \mathrm{O}_{4}$-dots and PEI-C/ $/ \mathrm{Fe}_{3} \mathrm{O}_{4}$-dots are compared with those of the precursors (FAC, $\mathrm{PEG}_{1500}$, and the PEI) in Fig. 4 \& 5. Overall, the spectra of the hybrid dots are apparently broadened, and both exhibit significant absorptions at $1770-1650 \mathrm{~cm}^{-1}$ due to carbonyl moieties, which are shifted from the carbonyl absorptions at $1650-1500 \mathrm{~cm}^{-1}$ in the spectrum of FAC (Fig. 4 \& 5). As known in the literature, ${ }^{\mathbf{4 3 , 4 4}}$ the strong carbonyl absorptions in neat citric acid are at $1770-1650 \mathrm{~cm}^{-1}$, and in FAC as a salt of citric acid with Fe(III), the absorptions of iron carboxylate are at $1650-1500 \mathrm{~cm}^{-1}$.

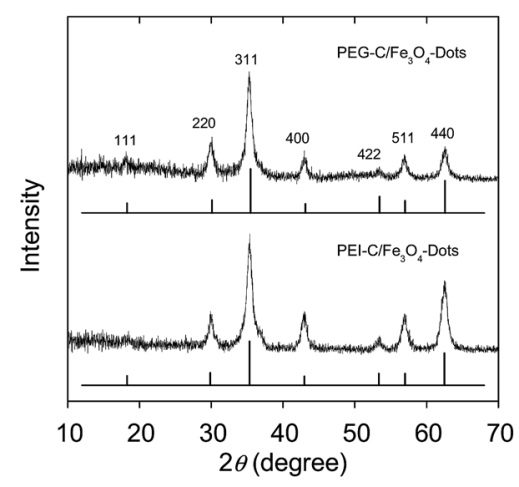

Fig. 2 Results from the X-ray powder diffraction characterization of the hybrid dots, with the standard diffraction pattern of cubic $\mathrm{Fe}_{3} \mathrm{O}_{4}$ also shown for comparison.

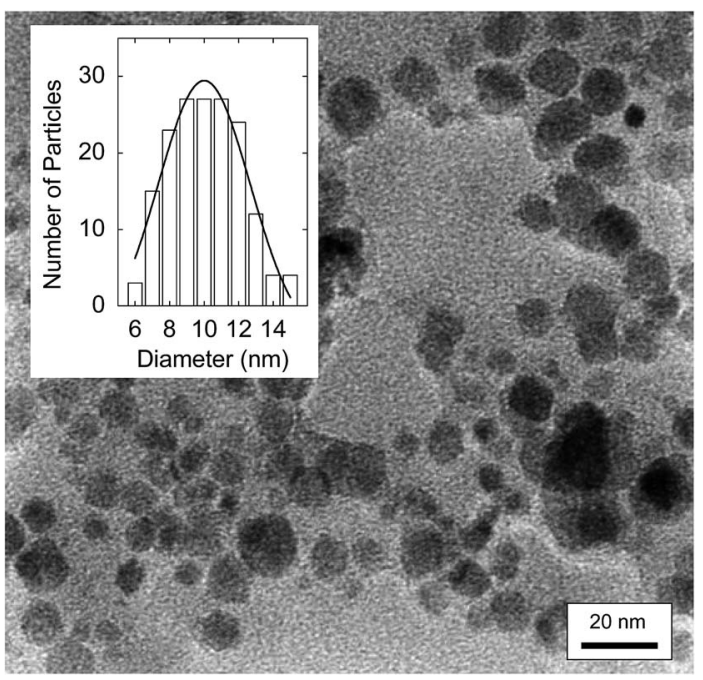

Fig. 3 A TEM image of the PEI-C/Fe $\mathrm{O}_{4}$-dots, with a statistical size analysis based on multiple images in the inset.

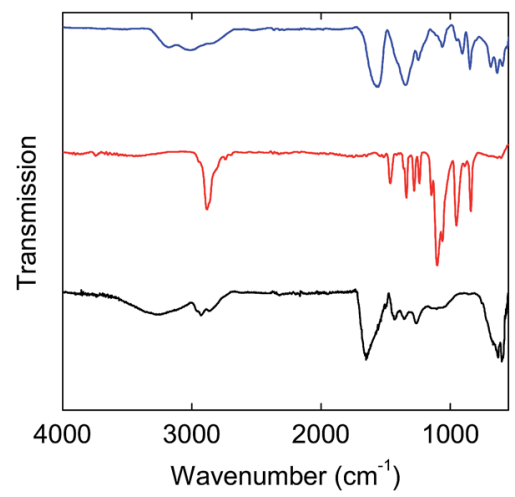

Fig. 4 FT-IR spectra of FAC (upper), $\mathrm{PEG}_{1500}$ (middle), and the PEG-C/ $\mathrm{Fe}_{3} \mathrm{O}_{4}$-dots (lower).

Thus, the thermal processing for the formation of the hybrid dots must have sacrificed the iron carboxylate in the precursor FAC to have some of the resulting citric acid-like species bound and/or trapped in the hybrid dot structures. For the PEG-C/ $\mathrm{Fe}_{3} \mathrm{O}_{4}$-dots, the characteristic $\mathrm{C}-\mathrm{H}$ absorptions at 2900-2700 $\mathrm{cm}^{-1}$ are much weaker than those in neat $\mathrm{PEG}_{1500}$, largely as expected for the PEGs being consumed in the carbonization processing. Similarly weakened $\mathrm{C}-\mathrm{H}$ absorptions are found in the spectrum of the PEI-C/Fe $\mathrm{O}_{4}$-dots. For both hybrid dots, the broad absorption bands at low frequencies (630-590 $\mathrm{cm}^{-1}$, Fig. 4 \& 5) may be assigned to $\mathrm{Fe}-\mathrm{O}$ bonds.

Aqueous dispersions of the hybrid dots exhibited more scattering in appearance visually and also in absorption spectral measurements even when the dispersions were very dilute, different from the solutions of neat CDots of similar surface functionalities reported previously. ${ }^{45}$ This might be due to several factors, including more significantly these hybrid dots being larger in size and heavier with a substantial content of $\mathrm{Fe}_{3} \mathrm{O}_{4}$. A commonly used strategy in improving the dispersion of inorganic and other nanoparticles ${ }^{\mathbf{4 6 , 4 7}}$ was adopted by adding 


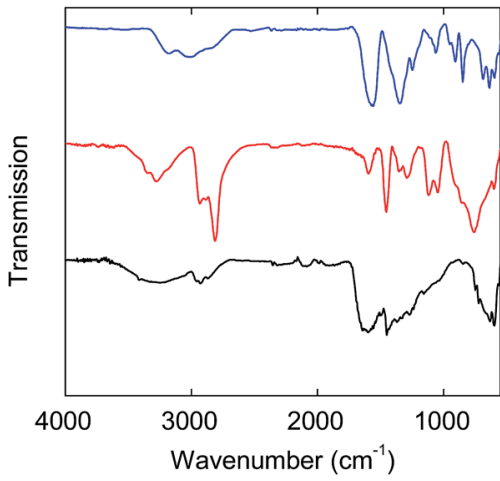

Fig. 5 FT-IR spectra of FAC (upper), the PEI (middle), and the PEI-C/ $\mathrm{Fe}_{3} \mathrm{O}_{4}$-dots (lower).

polyvinylpyrrolidone (PVP) polymers into the aqueous dispersions of the hybrid dots for reducing the light scattering in UV/ vis absorption measurements. As shown in Fig. 6, the observed absorption spectra of the aqueous dispersed PEG-C/ $/ \mathrm{Fe}_{3} \mathrm{O}_{4}$-dots and $\mathrm{PEI}-\mathrm{C} / \mathrm{Fe}_{3} \mathrm{O}_{4}$-dots with PVP polymers for improved dispersion are generally similar to that of the neat PEI-CDots reported previously. ${ }^{45}$ It should be pointed out that $\mathrm{Fe}_{3} \mathrm{O}_{4}$ nanoparticles are colored, but their absorptivities in the visible spectral region are generally low, ${ }^{48,49}$ and thus their contributions to the observed absorption spectra of the hybrid dots are insignificant.

Fluorescence spectra of the aqueous dispersed PEG-C/ $/ \mathrm{Fe}_{3} \mathrm{O}_{4^{-}}$ dots and PEI-C/ $/ \mathrm{Fe}_{3} \mathrm{O}_{4}$-dots are similar in profile to those of neat CDots, as illustrated in Fig. 6 for the comparison with the spectrum of neat PEI-CDots in aqueous solution, but their intensities or fluorescence quantum yields are lower. Between the two hybrid dots, the PEI-C/ $/ \mathrm{Fe}_{3} \mathrm{O}_{4}$-dots are significantly more fluorescent, consistent with the general observation in the literature that CDots of surface functionalization by amino molecules or polymers are higher in fluorescence quantum yields. ${ }^{14,15,50}$ Nevertheless, the observed quantum yields of the PEI-C/ $/ \mathrm{Fe}_{3} \mathrm{O}_{4}$-dots, on the order of $3 \%$ at $400 \mathrm{~nm}$ excitation, are still lower than those of the neat PEI-CDots (on average around

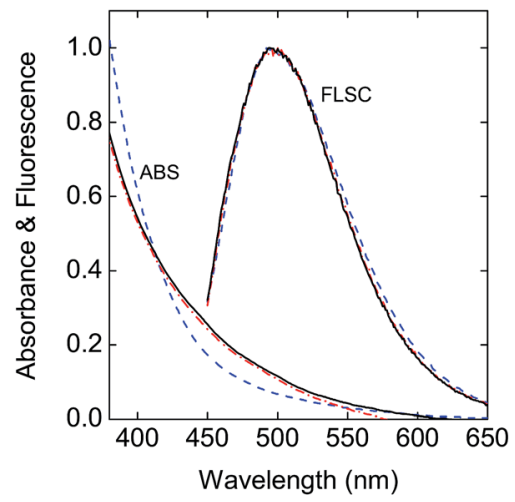

Fig. 6 Absorption (ABS) and fluorescence (FLSC, $440 \mathrm{~nm}$ excitation) spectra of the PEG-C/Fe $\mathrm{O}_{4}$-dots (-.-.-) and the PEI-C/Fe $\mathrm{O}_{4}$-dots $(-)$ in aqueous dispersions with PVP polymer as a dispersion agent are compared with those of the neat PEI-CDots in aqueous solution (- - - - $)^{45}$
$15 \%$ at the same excitation wavelength). ${ }^{45}$ Since the optical absorptions and fluorescence emissions in the visible spectral region are associated with the carbon domains in the hybrid dots, the observed lower fluorescence yields could be attributed to causes including the different formation processes and structural configurations of the hybrid dots from those of their neat CDots counterparts (resulting in less effective passivation of the carbon domains) and/or direct quenching effect of the $\mathrm{Fe}_{3} \mathrm{O}_{4}$ in the hybrid dots on the emissive excited states of the carbon domains. The latter as a possible cause was examined experimentally by removing $\mathrm{Fe}_{3} \mathrm{O}_{4}$ from the $\mathrm{PEI}-\mathrm{C} / \mathrm{Fe}_{3} \mathrm{O}_{4}$-dots, for which the dot sample was treated in an aqueous acid $(\mathrm{HCl})$ solution to dissolve the iron oxide, followed by dialysis to harvest the residual nanostructures of the hybrid dots without the $\mathrm{Fe}_{3} \mathrm{O}_{4}$ nanoparticles. For these $\mathrm{Fe}_{3} \mathrm{O}_{4}$-free residual "dots", their observed fluorescence spectra are largely the same as those of the original PEI-C/ $/ \mathrm{Fe}_{3} \mathrm{O}_{4}$-dots before the acid treatment (Fig. 7), again supporting the conclusion that the fluorescence emissions in the hybrid dots are due to the carbon domains passivated by the organic species. The fluorescence quantum yields of the hybrid dots post-acid treatment are around $4 \%$ at $400 \mathrm{~nm}$ excitation, somewhat higher than those of the same dots pre-acid treatment but still lower than those of the neat PEI-CDots reported previously. ${ }^{45}$ While there may be an argument that the comparison with the neat PEI-CDots is biased against the acid-treated hybrid dots because the acid treatment process may have other effects on the dot structures in addition to the removal of $\mathrm{Fe}_{3} \mathrm{O}_{4}$, such as effects that are negative to the effective passivation of the carbon domains by PEI and derived species in the residual dot structures post-acid treatment, it seems unlikely that the presence of $\mathrm{Fe}_{3} \mathrm{O}_{4}$ as a quencher within the hybrid dot structure could account entirely or predominantly for the observed lower fluorescence quantum yields of the hybrid dots. Therefore, it should be possible to prepare carbon $/ \mathrm{Fe}_{3} \mathrm{O}_{4}$ hybrid dots of competitive fluorescence performances to those of their neat CDots counterparts by developing more suitable synthetic strategies.

On the compositions and structures of individual hybrid dots, the average carbon content in the dot cores was estimated

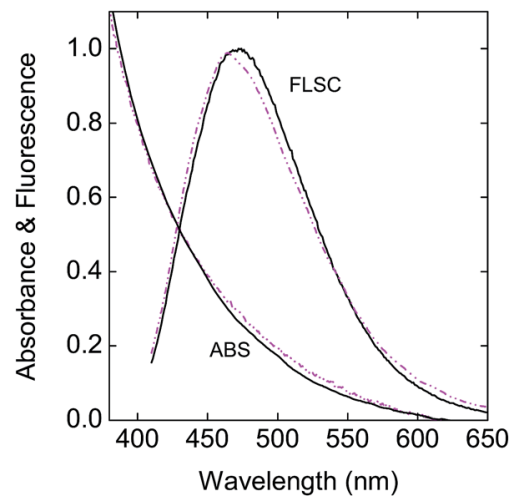

Fig. 7 Absorption (ABS) and fluorescence (FLSC, $400 \mathrm{~nm}$ excitation) spectra of the $\mathrm{PEI}-\mathrm{C} / \mathrm{Fe}_{3} \mathrm{O}_{4}$-dots before (-) and after (-..-..-) acid treatment in aqueous dispersions with and without PVP polymer, respectively. 
on the basis of the observed optical absorption in the visible spectrum by using the known absorptivities of small carbon nanoparticles. ${ }^{3}$ For the iron content, the classical colorimetric method based on the formation of red-colored iron(III) complex with thiocyanate was applied to the quantification by using a separately generated standard curve. ${ }^{51}$ The hybrid dots were first digested in established procedures before the colorimetric analyses. The estimated $\mathrm{C} / \mathrm{Fe}$ mass ratios in the hybrid dots were on the order of 0.5. According to the TEM imaging results, which are against the possibility of carbon nanoparticles and $\mathrm{Fe}_{3} \mathrm{O}_{4}$ nanoparticles in pairs or clusters, a more likely dot configuration is a $\mathrm{Fe}_{3} \mathrm{O}_{4}$ nanoparticle that is surface-coated with a layer of carbon and/or decorated with randomly distributed small carbon domains, which are functionalized by residual PEG (or PEI) species or segments (Fig. 1). The organic functionalization responsible for the passivation effect could be binding and/or the exposed segments of the organic species trapped in the dot surface structure. Such a hybrid dot configuration could be viewed in a first approximation as being on average a special structure of core and shells, namely the $\mathrm{Fe}_{3} \mathrm{O}_{4}$ nanoparticle core, a carbon shell (with an estimated thickness on the order of $1 \mathrm{~nm}$ ), and then a soft-shell (or corona) of bound or attached organic species (Fig. 1). Obviously, this structural view may be too simplified and idealized, as the actual arrangements of the carbon and $\mathrm{Fe}_{3} \mathrm{O}_{4}$ domains in the hybrid dots are probably more random and vary from dot to dot, for which a more accurate probing and understanding of structural details would require more sophisticated experimental techniques beyond the scope of this work.

The hybrid dots are obviously magnetic in terms of their response to external magnetic field in the magnetic separation. Similar magnetic response has been reported in the literature on other carbon- $\mathrm{Fe}_{3} \mathrm{O}_{4}$ combinations. . $^{24,32,33,52,53}$ Superparamagnetism in those materials has also been explored, ${ }^{52-55}$ so have been their uses as contrast agents in MRI experiments. ${ }^{32,33,52}$ While no dramatic differences are expected, an examination on the magnetic properties of the carbon $/ \mathrm{Fe}_{3} \mathrm{O}_{4}$ hybrid dots from this work against those already reported in the literature may prove interesting in further investigations.

\section{Conclusions}

Carbon/ $/ \mathrm{Fe}_{3} \mathrm{O}_{4}$ hybrid dots with oligomeric PEG and PEI species for surface functionalization and passivation could be synthesized in a facile thermal carbonization processing coupled with magnetic separation. The dot structure may be considered roughly as a small $\mathrm{Fe}_{3} \mathrm{O}_{4}$ nanoparticle (around $10 \mathrm{~nm}$ in diameter) coated or decorated with nanoscale carbon domains to which the organic species are attached via binding and/or other modes (some segments of the PEG or PEI survived the carbonization yet linked to the carbon domains in the hybrid dots, for example). The nanoscale carbon domains passivated by the organic species, which are similar to what are generally defined for CDots, are responsible for the observed fluorescence emissions of the hybrid dots. A major purpose of the study was to assess the potential quenching effect of $\mathrm{Fe}_{3} \mathrm{O}_{4}$ on the fluorescence emissions in the hybrid dots, and the results suggested that the quenching was hardly prohibitive. The observed lower fluorescence quantum yields of the hybrid dots than those of their neat counterparts were more likely due to changes in the synthesis of the dots, thus presenting opportunities for potentially developing synthesis methods that could produce carbon/ $\mathrm{Fe}_{3} \mathrm{O}_{4}$ hybrid dots of a much higher fluorescence performance. The combined fluorescence and magnetic properties in individual hybrid dots are amenable to applications that require both modalities, such as imaging and the sensing uses that couple magnetic separation with optical detection or quantification. To be more effective in these and other related applications, however, again further investigations aimed at a substantial enhancement of fluorescence properties in the hybrid dots are required. In addition to the dual modality uses (fluorescence/MRI as a more popular example), the hybrid dots may serve the purpose of carrying the magnetic moieties into organic or polymeric systems for various magnetic soft materials.

\section{Conflicts of interest}

There are no conflicts to declare.

\section{Acknowledgements}

Y. H. was a visiting student at Clemson University (CU) to perform most of the reported experiments, with a scholarship from the China Scholarship Council. The effort at CU was supported by the Air Force Office of Scientific Research (AFOSR) through the program of Dr Kenneth Caster. The support of National Key R\&D Program of China (2016YFA0202302, D. H.) is also acknowledged. L. R. T. and M. R. were participants of the Palmetto Academy funded and managed by the South Carolina Space Grant Consortium. S. Y. was a visiting student from Shantou University, China.

\section{References}

1 O. Kozák, M. Sudolská, G. Pramanik, P. Cígler, M. Otyepka and R. Zbořil, Chem. Mater., 2016, 28, 4085-4128.

2 L. Cao, M. J. Meziani, S. Sahu and Y.-P. Sun, Acc. Chem. Res., 2013, 46, 171-180.

3 J. Xu, S. Sahu, L. Cao, P. Anilkumsar, K. N. Tackett II, H. Qian, C. E. Bunker, E. A. Guliants, A. Parenzan and Y.-P. Sun, ChemPhysChem, 2011, 12, 3604-3608.

4 Y.-P. Sun, B. Zhou, Y. Lin, W. Wang, K. A. S. Fernando, P. Pathak, M. J. Meziani, B. A. Harruff, X. Wang, H. Wang, P. G. Luo, H. Yang, M. E. Kose, B. Chen, L. M. Veca and S.-Y. Xie, J. Am. Chem. Soc., 2006, 128, 7756-7757.

5 P. G. Luo, S. Sahu, S.-T. Yang, S. K. Sonkar, J. Wang, H. Wang, G. E. LeCroy, L. Cao and Y.-P. Sun, J. Mater. Chem. B, 2013, 1, 2116-2127.

6 K. Hola, Y. Zhang, Y. Wang, E. P. Giannelis, R. Zboril and A. L. Rogach, Nano Today, 2014, 9, 590-603.

7 Y. Wang and A. Hu, J. Mater. Chem. C, 2014, 2, 6921-6939. 
8 P. G. Luo, F. Yang, S.-T. Yang, S. K. Sonkar, L. Yang, J. J. Broglie, Y. Liu and Y.-P. Sun, RSC Adv., 2014, 4, 1079110807.

9 P. Miao, K. Han, Y. Tang, B. Wang, T. Lin and W. Cheng, Nanoscale, 2015, 7, 1586-1595.

10 K. A. S. Fernando, S. Sahu, Y. Liu, A. Jafariyan, W. K. Lewis, E. A. Guliants, A. Jafariyan, P. Wang, C. E. Bunker and Y.-P. Sun, ACS Appl. Mater. Interfaces, 2015, 7, 8363-8376.

11 S. Y. Lim, W. Shen and Z. Gao, Chem. Soc. Rev., 2015, 44, 362381.

12 Y. Du and S. Guo, Nanoscale, 2016, 8, 2532-2543.

13 G. E. LeCroy, S.-T. Yang, F. Yang, Y. Liu, K. A. S. Fernando, C. E. Bunker, Y. Hu, P. G. Luo and Y.-P. Sun, Coord. Chem. Rev., 2016, 320, 66-81.

14 X. Wang, L. Cao, S.-T. Yang, F. Lu, M. J. Meziani, L. Tian, K. W. Sun, M. A. Bloodgood and Y.-P. Sun, Angew. Chem., Int. Ed., 2010, 49, 5310-5314.

15 F. Yang, G. E. LeCroy, P. Wang, W. Liang, J. Chen, K. A. S. Fernando, C. E. Bunker, H. Qian and Y.-P. Sun, J. Phys. Chem. C, 2016, 120, 25604-25611.

16 Y.-P. Sun, X. Wang, F. Lu, L. Cao, M. J. Meziani, P. G. Luo, L. Gu and L. M. Veca, J. Phys. Chem. C, 2008, 112, 1829518298.

17 P. Anilkumar, X. Wang, L. Cao, S. Sahu, J.-H. Liu, P. Wang, K. Korch, K. N. Tackett II, A. Parenzan and Y.-P. Sun, Nanoscale, 2011, 3, 2023-2027.

18 J. Wang, S. Sahu, S. K. Sonkar, K. N. Tackett II, K. W. Sun, Y. Liu, H. Maimaiti, P. Anilkumar and Y.-P. Sun, RSC Adv., 2013, 3, 15604-15607.

19 J. Tian, Y. Leng, Z. Zhao, Y. Xia, Y. Sang, P. Hao, J. Zhan, M. Li and H. Liu, Nano Energy, 2015, 11, 419-427.

20 Y. Zhang, C. W. Foster, C. E. Banks, L. Shao, H. Hou, G. Zou, J. Chen, Z. Huang and X. Ji, Adv. Mater., 2016, 28, 9391-9399.

21 P. Chen, F. Wang, Z.-F. Chen, Q. Zhang, Y. Su, L. Shen, K. Yao, Y. Liu, Z. Cai, W. Lv and G. Liu, Appl. Catal., B, 2017, 204, 250-259.

22 L. Cao, S. Sahu, P. Anilkumar, C. E. Bunker, J. Xu, K. A. S. Fernando, P. Wang, E. A. Guliants, K. N. Tackett II and Y.-P. Sun, J. Am. Chem. Soc., 2011, 133, 4754-4757.

23 A. B. Bourlinos, A. Bakandritsos, A. Kouloumpis, D. Gournis, M. Krysmann, E. P. Giannelis, K. Polakova, K. Safarova, K. Hola and R. Zboril, J. Mater. Chem., 2012, 22, 23327.

24 H. Wang, Z. Wei, H. Matsui and S. Zhou, J. Mater. Chem. A, 2014, 2, 15740-15745.

25 Y. Zhang, Y. Shen, X. Teng, M. Yan, H. Bi and P. C. Morais, ACS Appl. Mater. Interfaces, 2015, 7, 10201-10212.

26 Y. Shi, Y. Pan, J. Zhong, J. Yang, J. Zheng, J. Cheng, R. Song and C. Yi, Carbon, 2015, 93, 742-750.

27 H. Wang, G. Cao, Z. Gai, K. Hong, P. Banerjee and S. Zhou, Nanoscale, 2015, 7, 7885-7895.

28 C. Han, H. Xu, R. Wang, K. Wang, Y. Dai, Q. Liu, M. Guo, J. Li and K. Xu, J. Mater. Chem. B, 2016, 4, 5798-5802.

29 S. Mohapatra, S. R. Rout, R. K. Das, S. Nayak and S. K. Ghosh, Langmuir, 2016, 32, 1611-1620.

30 F. Du, L. Zhang, L. Zhang, M. Zhang, A. Gong, Y. Tan, J. Miao, Y. Gong, M. Sun, H. Ju, C. Wu and S. Zou, Biomaterials, 2017, 121, 109-120.
31 Y. Pan, J. Yang, Y. Fang, J. Zheng, R. Song and C. Yi, J. Mater. Chem. B, 2017, 5, 92-101.

32 H. Wang, J. Shen, Y. Li, Z. Wei, G. Cao, Z. Gai, K. Hong, P. Banerjee and S. Zhou, Biomater. Sci., 2014, 2, 915-923.

33 B. Li, X. Wang, Y. Guo, A. Iqbal, Y. Dong, W. Li, W. Liu, W. Qin, S. Chen, X. Zhou and Y. Yang, Dalton Trans., 2016, 45, 5484-5491.

34 X. Huang, I. H. El-Sayed, W. Qian and M. A. El-Sayed, J. Am. Chem. Soc., 2006, 128, 2115-2120.

35 J. Yan, M. C. Estévez, J. E. Smith, K. Wang, X. He, L. Wang and W. Tan, Nano Today, 2007, 2, 44-50.

36 E. Boisselier and D. Astruc, Chem. Soc. Rev., 2009, 38, 17591782.

37 P. Zrazhevskiy, M. Sena and X. Gao, Chem. Soc. Rev., 2010, 39, 4326-4354.

38 E. Rampazzo, F. Boschi, S. Bonacchi, R. Juris, M. Montalti, N. Zaccheroni, L. Prodi, L. Calderan, B. Rossi, S. Becchi and A. Sbarbati, Nanoscale, 2012, 4, 824-830.

39 J. Li, W. Y. Lee, T. Wu, J. Xu, K. Zhang, G. Li, J. Xia and L. Bian, Adv. Healthcare Mater., 2016, 5, 1049-1057.

40 Y. Dong, R. Wang, H. Li, J. Shao, Y. Chi, X. Lin and G. Chen, Carbon, 2012, 50, 2810-2815.

41 S. Zhu, Q. Meng, L. Wang, J. Zhang, Y. Song, H. Jin, K. Zhang, H. Sun, H. Wang and B. Yang, Angew. Chem., Int. Ed., 2013, 52, 3953-3957.

42 Y. Liu, N. Xiao, N. Gong, H. Wang, X. Shi, W. Gu and L. Ye, Carbon, 2014, 68, 258-264.

43 M. Zhou, Z. Zhou, A. Gong, Y. Zhang and Q. Li, Talanta, 2015, 143, 107-113.

44 S. Lu, S. Guo, P. Xu, X. Li, Y. Zhao, W. Gu and M. Xue, Int. J. Nanomed., 2016, 11, 6325-6336.

45 Y. Hu, M. M. A. Awak, F. Yang, S. Yan, Q. Xiong, P. Wang, Y. Tang, L. Yang, G. E. LeCroy, X. Hou, C. E. Bunker, L. Xu, N. Tomlinson and Y.-P. Sun, J. Mater. Chem. C, 2016, 4, 10554-10561.

46 H. Wang, X. Qiao, J. Chen, X. Wang and S. Ding, Mater. Chem. Phys., 2005, 94, 449-453.

47 T. Romih, A. Jemec, M. Kos, S. B. Hocevar, S. Kralj, D. Makovec and D. Drobne, Environ. Pollut., 2016, 218, 957-964.

48 L. Wang, J. Luo, M. M. Maye, Q. Fan, Q. Rendeng, M. H. Engelhard, C. Wang, Y. Lin and C.-J. Zhong, J. Mater. Chem., 2005, 15, 1821-1832.

49 P. An, F. Zuo, X. Li, Y. Wu, J. Zhang, Z. Zheng, X. Ding and Y. Peng, Nano, 2013, 08, 1350061.

50 G. E. LeCroy, S. K. Sonkar, F. Yang, L. M. Veca, P. Wang, K. N. Tackett II, J.-J. Yu, E. Vasile, H. Qian, Y. Liu, P. G. Luo and Y.-P. Sun, ACS Nano, 2014, 8, 4522-4529.

51 J. Woods and M. Mellon, Ind. Eng. Chem., Anal. Ed., 1941, 13, 551-554.

52 H. Wang and S. Zhou, Biomater. Sci., 2016, 4, 1062-1073.

53 H. Wang, J. Shen, Y. Li, Z. Wei, G. Cao, Z. Gai, K. Hong, P. Banerjee and S. Zhou, ACS Appl. Mater. Interfaces, 2013, 5, 9446-9453.

54 Z. Wang, H. Guo, Y. Yu and N. He, J. Magn. Magn. Mater., 2006, 302, 397-404.

55 H. Wang, G. Cao, Z. Gai, K. Hong, P. Banerjee and S. Zhou, Nanoscale, 2015, 7, 7885-7895. 